\title{
METASTATIC CROHN'S DISEASE WITH ARTHRITIS AND WITHOUT INTESTINAL ACTIVITY: CASE REPORT
}

Severino Correia do Prado Neto ${ }^{1,2, *}$, Amanda Campos Souza Castro ${ }^{1}$, Brunna Albuquerque de Andrade ${ }^{1}$, Carolina Severiano de Miranda ${ }^{1}$, Leana Ferreira Crispim ${ }^{1}$

1. Faculdade Morgana Potrich, Mineiros (GO), Brazil. 2. Centro Universitário de Mineiros, Mineiros (GO), Brazil.

*Corresponding author: severinopradoneto@gmail.com

\section{BACKGROUND}

Crohn's disease (CD) is a chronic idiopathic inflammatory disease that can affect the entire gastrointestinal tract, with bulging focal lesions and transmural inflammation, interspersing periods of remission and crises. The annual incidence is 5 to 50 cases per 100,000 inhabitants. In some cases, extraintestinal manifestations (EIMs) can also occur, including dermatological (2 to 34\%) and rheumatological (2.8 to $62 \%$ ), the latter being the most common EIM. The presence of concomitant EIMs is less frequent (4.5 to $14.6 \%$ of cases). Metastatic Crohn's disease (MCD) is a rare skin manifestation, described in series and case reports. It is characterized by lesions in various locations of the skin, with a predilection for genitals, limbs and fold regions, and large areas of healthy skin are separated from intestinal lesions. In adults, MCD usually appears after diagnosis of the intestinal condition. Only $20 \%$ of patients have MCD without any previous intestinal manifestation. In MCD, involvement of different locations at the same time is rare (less than $1 \%$ ). The presence of noncaseating granulomas in skin biopsies is suggestive of MCD.

\section{CASE REPORT}

A 53-year-old woman diagnosed with inflammatory bowel disease in 2017 during a colonoscopy evaluation due to dyspareunia, without intestinal symptoms. She showed partial improvement with mesalazine. In 2019, she developed multiple ulcerated and painful lesions in the armpits (Fig. 1), trunk and limbs, in addition to arthritis in the ankles and knees, but without gastrointestinal complaints. Complementary exam results: blood count, TGO, TGP, urea, creatinine, normal uric acid; elevated PCR and ESR; FAN, FR, anti-CCP, HIP negative; HLA B-27 not detected; HIV, VDRL, hepatitis B and C, research for Leishmania and BAAR in the negative lymph. Biopsy compatible with noncaseous granulomatous dermatitis, with a marked diffuse mononuclear inflammatory infiltrate, composed of lymphocytes, plasmocytes, histiocytes, with some intervening neutrophils and granuloma sketches, with research of BAAR, Leishmania and negative fungi, without signs of malignancy. Metastatic Crohn's disease was diagnosed with arthritis and without intestinal activity and prednisone $40 \mathrm{mg} /$ day and sulfasalazine were started. In reevaluation, she presented clinical (Fig. 2) and laboratory improvement, weaning from corticotherapy. Report after the patient's consent.

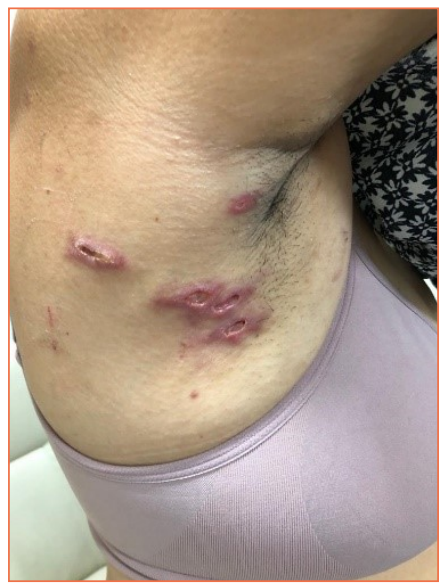

Figure 1. Armpit ulcers 


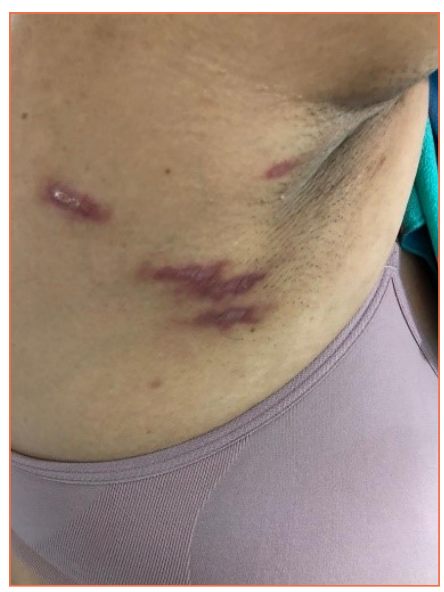

Figure 2. Healing armpit ulcers

\section{CONCLUSION}

This is an atypical case of $C D$ both from the intestinal point of view and from the EIMs, highlighting the importance of MCD in the spectrum of different diagnoses of granulomatous skin diseases. 